\title{
Chiral Bosons Through Linear Constraints
}

\author{
H. O. Girottit, M. Gomes and V. O. Rivelles \\ Instituto de Física, Universidade de São Paulo, \\ Caixa Postal 20516, 01498 São Paulo, SP, Brazil.
}

\begin{abstract}
We study in detail the quantization of a model which apparently describes chiral bosons. The model is based on the idea that the chiral condition could be implemented through a linear constraint. We show that the space of states is of indefinite metric. We cure this disease by introducing ghost fields in such a way that a BRST symmetry is generated. A quartet algebra is seen to emerge. The quartet mechanism, then, forces all physical states, but the vacuum, to have zero norm.
\end{abstract}

11.10.Ef, 03.70.+k

Typeset Using REVTEX 
It has been claimed in the literature [1,2] that the two dimensional Lorentz invariant model [3]

$$
\mathcal{L}=\frac{1}{2}\left(\partial_{\mu} \varphi\right)\left(\partial^{\mu} \varphi\right)+\lambda_{\mu}\left(g^{\mu \nu}-\epsilon^{\mu \nu}\right) \partial_{\nu} \varphi
$$

describes chiral bosons, namely, a field satisfying the equation $\partial_{-} \varphi \equiv\left(\partial_{0}-\partial_{1}\right) \varphi=0$. The procedure for constructing the Lagrangian (11) is rather obvious, the chiral condition has been "linearly" added to the Lagrangian of a free massless scalar field through the Lagrange multiplier $\lambda_{\mu}$. However, from the equations of motion deriving from (四),

$$
\begin{aligned}
& \partial_{\mu} \partial^{\mu} \varphi+\left(g^{\mu \nu}-\epsilon^{\mu \nu}\right) \partial_{\nu} \lambda_{\mu}=0, \\
& \left(g^{\mu \nu}-\epsilon^{\mu \nu}\right) \partial_{\nu} \varphi=0 \\
& \left(g^{\mu \nu}-\epsilon^{\mu \nu}\right) \partial_{\nu} \lambda_{\mu}=0
\end{aligned}
$$

one sees that not only $\varphi$ but also $\lambda_{\mu}$ are chiral fields. The fact that the Lagrange multiplier $\lambda_{\mu}$ becomes dynamical was first noticed by Siegel [4].

Within the Hamiltonian formulation, the model is specified by the canonical Hamiltonian

$$
H_{0}=\int d x \Pi(x) \varphi^{\prime}(x)
$$

together with the second class constraints

$$
\begin{aligned}
& T_{1}(x) \equiv p_{\lambda}(x) \approx 0, \\
& T_{2}(x) \equiv \lambda_{+}(x)-\Pi(x)+\varphi^{\prime}(x) \approx 0,
\end{aligned}
$$

where $\Pi$ and $p_{\lambda}$ are the canonical conjugate momenta of $\varphi$ and $\lambda_{+} \equiv \lambda_{0}+\lambda_{1}$, respectively. Furthermore, $\varphi^{\prime}(\dot{\varphi})$ is a shorthand notation for $\partial_{1} \varphi\left(\partial_{0} \varphi\right)$. The above constraints allow for the elimination of the sector $\lambda_{+}, p_{\lambda}$ from the phase space. The reduced phase space is then spanned by the variables $\varphi$ and $\Pi$ whose Dirac brackets [5] are, as they must [7], equal to the corresponding Poisson brackets. Hence, when formally quantized according to the Dirac bracket procedure [5], the theory appears to describe a single chiral field [1]. 
In this paper we study in detail the particle content of the model. As we shall see, the metric of the space of states is not positive definite. We cure this problem by adding ghosts to the original Lagrangian so that a BRST symmetry emerges. We demonstrate, afterwards, that the original fields and the ghosts obey a quartet algebra [6]. Then, the quartet mechanism [6], when applied to this case, leads to the conclusion that the only surviving state of positive norm is the vacuum state. Thus the model is not appropriate to describe neither chiral bosons nor any other quantum excitation.

As pointed out in ref. [1], the quantum equations of motion obeyed by the fields $\varphi$ and $\Pi$ are $\partial_{-} \varphi=0$ and $\partial_{-} \Pi=0$. These equations and the canonical equal-time commutation relations are solved by $\left(x^{+} \equiv x^{0}+x^{1}\right)$

$$
\begin{aligned}
& \varphi\left(x^{+}\right)=\frac{1}{\sqrt{2 \pi}} \int_{0}^{\infty} d p\left[\mathrm{e}^{-i p x^{+}} a(p)+\mathrm{e}^{i p x^{+}} a^{\dagger}(p)\right], \\
& \Pi\left(x^{+}\right)=\frac{1}{\sqrt{2 \pi}} \int_{0}^{\infty} d p\left[\mathrm{e}^{-i p x^{+}} b(p)+\mathrm{e}^{i p x^{+}} b^{\dagger}(p)\right]
\end{aligned}
$$

with

$$
\left[a(p), b^{\dagger}\left(p^{\prime}\right)\right]=-\left[b(p), a^{\dagger}\left(p^{\prime}\right)\right]=i \delta\left(p-p^{\prime}\right)
$$

as the only nonvanishing commutators.

The normal ordered quantum counterpart of the classical Hamiltonian $H_{0}$ is

$$
H_{0}=i \int_{0}^{\infty} d p p\left[a^{\dagger}(p) b(p)-b^{\dagger}(p) a(p)\right]
$$

To make explicit that the space of states we are dealing with is of indefinite metric, we introduce the operators

$$
\begin{aligned}
A & \equiv \frac{1}{\sqrt{2}}(a+i b) \\
B & \equiv \frac{1}{\sqrt{2}}(a-i b),
\end{aligned}
$$

which are easily seen to obey the commutation relations

$$
\left[A(p), A^{\dagger}\left(p^{\prime}\right)\right]=-\left[B(p), B^{\dagger}\left(p^{\prime}\right)\right]=\delta\left(p-p^{\prime}\right) .
$$


It is now clear that all states obtained by applying to the vacuum the operator $B^{\dagger}$ an odd number of times are of negative norm. In terms of $A$ and $B$ the Hamiltonian assumes the standard form

$$
H_{0}=\int_{0}^{\infty} d p p\left[A^{\dagger}(p) A(p)-B^{\dagger}(p) B(p)\right]
$$

The lack of boundedness of $H_{0}$ at the classical level reflects itself, at the quantum level, through the appearance of states of negative norm.

To cure the disease represented by the states of negative norm, we bring into the theory the real Grassmann fields $\bar{C}_{\mu}(x)$ and $C(x)$. This is done by adding to $\mathcal{L}$ the ghost Lagrangian

$$
\mathcal{L}_{g}=i C \partial_{-} \bar{C}_{+},
$$

where $\bar{C}_{+} \equiv \bar{C}_{0}+\bar{C}_{1}$. One can corroborate that $\mathcal{L}_{T} \equiv \mathcal{L}+\mathcal{L}_{g}$ is invariant under the global nilpotent transformation

$$
\begin{aligned}
\delta \varphi(x) & =i \epsilon_{-} \bar{C}_{+}(x) \\
\delta \lambda_{+}(x) & =i \epsilon_{-} \partial_{+} \bar{C}_{+}(x) \\
\delta \bar{C}_{+}(x) & =0 \\
\delta C(x) & =-i \epsilon_{-} \lambda_{+}(x)-\frac{i}{2} \epsilon_{-} \partial_{+} \varphi(x) .
\end{aligned}
$$

We emphasize that the original bosonic Lagrangian does not posses a local symmetry since it only exhibits second class constraints. Nevertheless, a BRST symmetry has emerged after the addition of the ghost fields. An analogous situation has already been encountered in the literature [8].

The canonical ghost Hamiltonian

$$
H_{0}^{g}=i \int d x C(x) \bar{C}_{+}^{\prime}(x)
$$

and the second class constraints

$$
\begin{aligned}
& T_{1}^{g}(x) \equiv p(x) \approx 0, \\
& T_{2}^{g}(x) \equiv \bar{p}_{-}(x)-i C(x) \approx 0
\end{aligned}
$$


define the dynamics of the ghost fields in the Hamiltonian framework. Here, $p$ and $\bar{p}_{-}$are the canonical conjugate momenta of $C$ and $\bar{C}_{+}$, respectively. Clearly, the sector $C, p$ can be be eliminated from phase space, although, following common practice, we shall keep $\bar{C}_{+}$ and $C$ as the canonical variables spanning the ghost sector of the reduced phase space. As required [7], the Dirac brackets involving $\bar{C}_{+}$and $C$ equal the corresponding generalized Poisson brackets. The quantum counterpart of $H_{0}^{g}$ is obtained from (15) after appropriate symmetrization, required to solve the ordering problem. The equations of motion obeyed by the ghost field operators are, then, found to be $\partial_{-} C=0$ and $\partial_{-} \bar{C}_{+}=0$. These equations and the canonical equal-time anticommutation relations are solved by

$$
\begin{aligned}
& C\left(x^{+}\right)=\frac{1}{\sqrt{2 \pi}} \int_{0}^{\infty} d p\left[\mathrm{e}^{-i p x^{+}} d(p)+\mathrm{e}^{i p x^{+}} d^{\dagger}(p)\right] \\
& \bar{C}_{+}\left(x^{+}\right)=\frac{1}{\sqrt{2 \pi}} \int_{0}^{\infty} d p\left[\mathrm{e}^{-i p x^{+}} \bar{d}(p)+\mathrm{e}^{i p x^{+}} \bar{d}^{\dagger}(p)\right]
\end{aligned}
$$

where the nonvanishing anticommutators are

$$
\left\{d(p), \bar{d}^{\dagger}\left(p^{\prime}\right)\right\}=\left\{d^{\dagger}(p), \bar{d}\left(p^{\prime}\right)\right\}=\delta\left(p-p^{\prime}\right)
$$

By replacing (17) and (18) in $H_{0}^{g}$ one arrives to

$$
H_{0}^{g}=\int_{0}^{\infty} d p p\left[\bar{d}^{\dagger}(p) d(p)+d^{\dagger}(p) \bar{d}(p)\right]
$$

where the normal ordering prescription has been used. After attributing ghost number -1 and +1 to $C$ and $\bar{C}_{+}$, respectively, one finds that

$$
i N_{g}=\int_{0}^{\infty} d p\left[\bar{d}^{\dagger}(p) d(p)-d^{\dagger}(p) \bar{d}(p)\right]
$$

where $N_{g}$ denotes the hermitean ghost number operator.

Our next step consists in constructing the BRST charge operator. One can verify that

$$
Q \equiv-\int_{0}^{\infty} d p\left[\bar{d}^{\dagger}(p) b(p)+b^{\dagger}(p) \bar{d}(p)\right]
$$

correctly implements the quantum analog of the global transformation (14). Furthermore, $Q^{2}=0$. By using the commutation relations (7) and (19) one arrives to the quartet algebra 66 


$$
\begin{gathered}
{[Q, a(p)]=i \bar{d}(p),} \\
{[Q, b(p)]=0,} \\
\{Q, \bar{d}(p)\}=0, \\
\{Q, d(p)\}=-b(p) .
\end{gathered}
$$

We now recall that physical states are required to verify $Q \mid$ phys $>=0$. Hence, by the quartet mechanism [6], all physical states, with the exception of the vacuum, are zero-norm states. The physical S-matrix is just the identity operator and $<0\left|H_{0}+H_{0}^{g}\right| 0>=0$.

Thus, the addition of ghosts render the theory consistent but, however, trivial. We then conclude that linear constraints do not provide an efficient mechanism to generate chiral bosons. We mention that several models for chiral bosons not based on the linear constraint, and therefore free of the above difficulties, have been proposed in the past 4.9611 .

\section{ACKNOWLEDGMENTS}

This work has been supported in part by Conselho Nacional de Desenvolvimento Científico e Tecnológico (CNPq), Brazil, and Fundação de Amparo à Pesquisa do Estado de São Paulo (FAPESP), Brazil. 


\section{REFERENCES}

* On leave of absence from Instituto de Física, Universidade Federal do Rio Grande do Sul, 91500 Porto Alegre, RS, Brazil.

[1] P. P. Srivastava, Phys. Rev. Lett. 63, 2791 (1989).

[2] D. S. Kulshreshtha and H. J. W. Müller-Kirsten, Phys Rev. 45, 393 (1992).

[3] In this paper we use the Minkowski metric $g^{00}=-g^{11}=1, g^{\mu \nu}=0$ if $\mu \neq \nu$. Also $\epsilon^{\mu \nu}=-\epsilon^{\nu \mu}, \epsilon^{01}=1$.

[4] W. Siegel, Nucl. Phys. B238, 307 (1984).

[5] P. A. M. Dirac, Lectures on Quantum Mechanics, Belfer Graduate School of Science (Yeshiva Univ. Press, New York, 1964); Can. J. Math. 2, 129 (1950).

[6] T. Kugo and I. Ojima, Suppl. Prog. Theor. Phys. 66, 1 (1979).

[7] E. S. Fradkin and G. A. Vilkovisky, CERN report TH. 2332 (1977), unpublished.

[8] T. Kashiwa, Prog. Theor. Phys. 70, 1124 (1983).

[9] J. Labastida and M. Pernici, Phys. Rev. Lett. 59, 2511 (1987); Nucl. Phys. B297 557, (1988); C. Imbimbo and A. Schwimmer, Phys. Lett. 193B, 455 (1987); M. Mezincescu and R. I. Nepomechie, Phys. Rev. D37, 3067 (1988); M. Bernstein and J. Sonnenschein, Phys. Rev. Lett. 60, 1772 (1988).

[10] R. Floreanini and R. Jackiw, Phys. Rev. Lett. 59, 1873 (1987); L. Faddeev and R. Jackiw, Phys. Rev. Lett. 60, 1692 (1988);

[11] M. E. V. Costa and H. O. Girotti, Phys. Rev. Lett. 60, 1771 (1988); H. O. Girotti, M. Gomes, V. Kurak, V. O. Rivelles and A. J. da Silva ibid. 60, 1913 (1988); H. O. Girotti, M. Gomes, V. O. Rivelles and A. J. da Silva, Phys. Rev. D39, 3792 (1989). 